\title{
UNIVERSITY OF MIAMI RADIOCARBON DATES XVI
}

\section{S INTRONE, R JOHNSON, and J J STIPP}

Department of Geology, University of Miami, Coral Gables, Florida 33124

Radiocarbon measurements have been continued on a variety of projects and materials. Chemical and counting procedures remain the same as indicated in R, v 20, p 274-282. Dates are calculated using the Libby ${ }^{14} \mathrm{C}$ half-life of 5568 years; errors are reported as one-standard deviation $(1 \sigma)$ based only on statistical counting uncertainties in background, modern standard, and sample activities. All samples for which ${ }^{13} \mathrm{C} /{ }^{12} \mathrm{C}$ ratios are available are corrected for isotopic fractionation by normalizing to $-25 \%$. A 400-year reservoir age correction has been applied to marine carbonates.

\section{SAMPLE DESCRIPTIONS}

I. GEOLOGIC SAMPLES

\section{Cape Cod series}

\section{A. United States}

Peat samples from bog and salt marsh were dated to determine development of spit and rate of barrier migration in Cape Cod, Massachusetts. Coll and subm 1979 by S Leatherman, Univ Massachusetts, Amherst.

\section{UM-1592. PL-3-26-10T}

$$
\begin{array}{r}
890 \pm 80 \\
590 \pm 120 \\
670 \pm 70 \\
540 \pm 120
\end{array}
$$$$
\text { UM-1593. PL-4-3B-3 }
$$

UM-1595. PL-2-32-5

UM-1596. PL-1-25-6

The 4 samples above are all bog peats buried by eolian sands from Provincetown, Massachusetts $\left(42^{\circ} 05^{\prime} \mathrm{N}, 70^{\circ} 11^{\prime} \mathrm{W}\right)$.

UM-1594. C-3-60

$$
810 \pm 100
$$

Salt marsh peat from Nauset spit, Massachusetts $\left(41^{\circ} 50^{\prime} 0^{\prime \prime} \mathrm{N}, 69^{\circ}\right.$ $\left.56^{\prime} \mathrm{W}\right)$. Sample is from behind barrier beach and buried by overwash deposits.

\section{Cape Romain series}

Several cores from Cape Roman, South Carolina have yielded assorted shell, plant root and peat samples dated for stratigraphic reconstruction of barrier island and its correlation to sea level. Coll and subm by Ci Ruby Sept 1978, Univ South Carolina, Columbia.

\section{UM-1626. CRT1-21-C}

$$
\begin{array}{r}
6600 \pm 160 \\
\delta^{1.3} C=-27.29 \%
\end{array}
$$

Plant roots that penetrate muddy-sand with shell fragments $\left(79^{\circ} 20^{\prime}\right.$ $00^{\prime \prime} \mathrm{N}, 33^{\circ} 03^{\prime} 00^{\prime \prime} \mathrm{W}$ ) indicating possible relict back barrier beach. 
UM-1627. CRT3-9-D

$14,400 \pm 170$

$\delta^{13} \mathrm{C}=-25.73 \%$

Basal peat (probably fresh water) overlain by brackish and salt water facies $\left(79^{\circ} 21^{\prime} 00^{\prime \prime} \mathrm{N}, 33^{\circ} 03^{\prime} 00^{\prime \prime} \mathrm{W}\right)$.

UM-1628. CRT1-8-H

$4410 \pm 110$

$\delta^{13} C=-0.47 \%$ W).

Shell storm lag overlain by bay fill muds $\left(79^{\circ} 21^{\prime} 00^{\prime \prime} \mathrm{N}, 33^{\circ} 01^{\prime} 30^{\prime \prime}\right.$

UM-1629. CRT1-15-D

$10,360 \pm 170$

Basal peat overlain by later brackish and salt water deposits $\left(79^{\circ} 22^{\prime}\right.$ $\left.00^{\prime \prime} \mathrm{N}, 33^{\circ} 01^{\prime} 30^{\prime \prime} \mathrm{W}\right)$.

\section{Kiawah and Seabrook Islands series}

Samples of shell, wood, and peat coll from Kiawah and Seabrook Is, South Carolina. Dated for construction of stratigraphic time lines for determination of depositional history and sea level fluctuations. Coll June 1978 and subm by T Moslow, Univ South Carolina, Columbia.

UM-1539. SI-8 : 4-C

$4230 \pm 80$

Shell material (Crassostrea virginica) taken at depth $11 \mathrm{~m}$ in silty clay (salt marsh depositional environment) in Seabrook I. (32 $34^{\prime} 05^{\prime \prime} \mathrm{N}, 80^{\circ}$ $\left.11^{\prime} 01^{\prime \prime} \mathrm{W}\right)$.

UM-1540. KI-1 : 9A

$1370 \pm 60$

Predominantly Mulinia lateralis taken from thinly laminated silty sand to silty clay assoc with nearshore marine depositional environment. Sample at base of regressive sequence at depth $8 \mathrm{~m}$, Kiawah I. $\left(32^{\circ} 36^{\prime} 15^{\prime \prime}\right.$ $\mathrm{N}, 80^{\circ} 04^{\prime} 15^{\prime \prime} \mathrm{W}$ ).

UM-154.1. SI-7 : 8-B

$1650 \pm 130$

Wood sample from Seabrook I. at depth $5 \mathrm{~m}\left(32^{\circ} 34^{\prime} 10^{\prime \prime} \mathrm{N}, 80^{\circ} 10^{\prime}\right.$ $\left.45^{\prime \prime} \mathrm{W}\right)$. Found in interbedded, burrowed, silty sand and clay assoc with tidal flat or marsh depositional environment.

UM-1599. KI-2:25' to $27^{\prime}$

$$
\begin{array}{r}
\mathbf{2 7 1 0} \pm \mathbf{8 0} \\
\delta^{13} \mathrm{C}=+0.50 \% \text { o }
\end{array}
$$

Shell material, predominantly Mulinia occurring in layers of silty sand on Kiawah I. $\left(32^{\circ} 36^{\prime} 25^{\prime \prime} \mathrm{N}, 80^{\circ} 04^{\prime} 20^{\prime \prime} \mathrm{W}\right)$.

UM-1600. KI-2:30' to $31^{\prime}$

$$
\begin{array}{r}
4450 \pm \mathbf{8 0} \\
\delta^{1.3} C=+0.05 \%
\end{array}
$$

Predominantly Mulinia in silty clay matrix, basal Holocene sediments on Kiawah I. $\left(32^{\circ} 36^{\prime} 25^{\prime \prime} \mathrm{N}, 80^{\circ} 04^{\prime} 20^{\prime \prime} \mathrm{W}\right)$.

\section{UM-1601. KI-4:20' to $22^{\prime}$}

$$
\begin{array}{r}
\mathbf{3 6 4 0} \pm \mathbf{8 0} \\
\delta^{13} C=-0.25 \%
\end{array}
$$

Primarily Mulinia in layers in interbedded silty sand and silty clay assoc with Holocene shoreface on Kiawah I. $\left(32^{\circ} 36^{\prime} 50^{\prime \prime} \mathrm{N}, 80^{\circ} 04^{\prime} 05^{\prime \prime}\right.$ W). 
UM-1602. KI-7 :-10.5'

Wood, believed to be root or trunk portion of climax maritime forest tree (Kiawah) ( $\left.92^{\circ} 36^{\prime} 55^{\prime \prime} \mathrm{N}, 80^{\circ} 05^{\prime} 18^{\prime \prime} \mathrm{W}\right)$.

UM-1603. SI-9:26' to $28^{\prime}$

$$
\begin{gathered}
\mathbf{2 2 , 7 7 0} \\
\delta^{1 .} C=-16.93 \% \\
-\mathbf{5 5 0}
\end{gathered}
$$

Peat, probably rafted and Spartina roots in compact marsh clay (Seabrook) $\left(32^{\circ} 34^{\prime} 55^{\prime \prime} \mathrm{N}, 80^{\circ} 09^{\prime} 10^{\prime \prime} \mathrm{W}\right)$. Sample taken at base of postulated Holocene sec.

\section{Kiawah Island series}

Samples of whole and fragmental shell material predominantly Mulinia lateralis coll from Kiawah I., South Carolina. Dated to determine age of depositional event in back barrier of Kiawah. Coll 1978 and subm by A Duc, Univ South Carolina, Columbia.

\section{UM-1590. KIV $11: 12$}

$$
\begin{array}{r}
\mathbf{4 4 2 0} \pm \mathbf{8 0} \\
\delta^{13} C=-0.65 \%
\end{array}
$$

Sample from near base of Holocene sec 3.5 to $3.9 \mathrm{~m}$ depth $\left(32^{\circ} 36^{\prime} 58^{\prime \prime}\right.$ $\left.\mathrm{N}, 80^{\circ} 04^{\prime} 22^{\prime \prime} \mathrm{W}\right)$. Specifically from storm episode unconformably overlying marsh deposits.

\section{UM-1591. NOI 2:16}

$$
\begin{array}{r}
31,160+660 \\
\delta^{13} C=+0.19 \%
\end{array}
$$

Sample below ca $2 \mathrm{~m}$ green clay interpreted as Pleistocene $\left(32^{\circ} 36^{\prime}\right.$ $\left.40^{\prime \prime} \mathrm{N}, 80^{\circ} 07^{\prime} 15^{\prime \prime} \mathrm{W}\right)$. Sample depth 4.8 to $5.1 \mathrm{~m}$.

\section{Baltimore Canyon series}

Carbonaceous clays from continental slope were dated to establish sedimentation rate and to correlate with other cores and dates. Cores $14 \mathrm{~B}$ $\left(37.53^{\circ} \mathrm{N}, 73.53^{\circ} \mathrm{W}\right) 14 \mathrm{E}\left(37.25^{\circ} \mathrm{N}, 73.14^{\circ} \mathrm{W}\right)$ and $14 \mathrm{D}\left(37.43^{\circ} \mathrm{N}, 73.36^{\circ}\right.$ $\mathrm{W})$ are piston cores taken on continental slope off $\mathrm{E}$ United States coast in vicinity of Baltimore Canyon. Coll March 1975 by G Hayward; subm by L Doyle, Univ South Florida, St Petersburg.

UM-1535. 14B-210 to $240 \mathrm{~cm}$

UM-1536. 14D-45 to $65 \mathrm{~cm}$

UM-1537. 14D.80 to $100 \mathrm{~cm}$

$$
\begin{array}{r}
7180 \pm 120 \\
+470 \\
25,900 \quad-440 \\
+1050 \\
29,650 \quad-930
\end{array}
$$




\section{UM-1538. 14E-260 to $280 \mathrm{~cm}$}

\section{B. Bermuda}

\section{Bermuda Lagoon series}

Series of cores and coral samples coll 1979 by P Garrett and E Shinn in Bermuda were dated to reconstruct reef history and measure rate of reef growth. Subm by P Garrett, Univ California, Santa Barbara.

UM-1604. $7 \mathrm{~m} / 1115-19 \quad 3900 \pm 70$

Diploria from South Olympic reef $2.7 \mathrm{~km}$ due $\mathrm{S}$ of North Rock in Bermuda Lagoon $\left(32^{\circ} 26^{\prime} 52^{\prime \prime} \mathrm{N}, 64^{\circ} 46^{\prime} 20^{\prime \prime} \mathrm{W}\right)$.

UM-1605. $7 \mathrm{~m} / 1247-0$

$$
1760 \pm 80
$$

Montastrea annularis.

UM-1606. $7 \mathrm{~m} / 1247-9$

Montastrea annularis.

UM-1607. $7 \mathrm{~m} / 1247-32$

$$
\mathbf{3 0 0 0} \pm \mathbf{8 0}
$$

Coralline sp unknown.

UM-1608. $7 \mathrm{~m} / 1247-40$

$$
4930 \pm 90
$$

Montastrea cavernosa.

General Comment: UM-1605 -1608 are from Bermuda lagoon (32 $26^{\prime} 11^{\prime \prime}$ N, $64^{\circ} 49^{\prime} 35^{\prime \prime}$ W) $13.5 \mathrm{~N}$ of Dundonald Channel.

UM-1609. $4 \mathrm{~m} / 1013-0$

$220 \pm 70$

Diploria.

UM-1610. $4 \mathrm{~m} / 1013-8$

$1930 \pm 70$

Diploria labyrinthiformis.

UM-1611. 4m/1013-16

$2820 \pm 110$

Montastrea cavernora.

UM-1612. 4m/1013-20

$$
3790 \pm 80
$$

Diploria strigosa.

UM-1613. 4m/1013-30

$3640 \pm 90$

Diploria strigosa.

General Comment: UM-1609 -1613 are $2 \mathrm{~km} \mathrm{~N}$ of Blue Cut $\left(32^{\circ} 24^{\prime} 30^{\prime \prime}\right.$ $\mathrm{N}, 64^{\circ} 52^{\prime} 40^{\prime \prime} \mathrm{W}$ ) on NW Bermuda Ledge Flats.

UM-1614. 4m/1805-1

$4530 \pm 90$

Several sp of shells.

UM-1615. 4m/1805-15

$2590 \pm 90$

Chama macrophyllo. 
General Comment: UM-1614 and UM-1615 are $1 \mathrm{~km}$ NW of Bailey's Bay

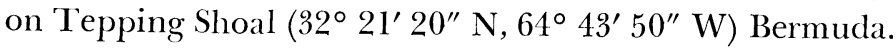

UM-1616. DSE-5

Diploria Labyrintheformis.

$3060 \pm 70$

UM-1617. DSE-24.

Diploria Labyrintheformis.

$4130 \pm 80$

UM-1618. DSE-52

Montastrea cavernosa.

General Comment: UM-1616 -1618 are W of Daniels I. $\left(32^{\circ} 18^{\prime} 25^{\prime \prime} \mathrm{N}\right.$, $\left.64^{\circ} 53^{\prime} 12^{\prime \prime} \mathrm{W}\right)$.

\section{South America}

\section{Argentina Coastal series}

Assorted shell samples from coastal area of Punta Medana. Shells dated to reconstruct stratigraphy. Coll by S Parker Oct 1977; subm by S Parker, Servicia Hidrografia Naval, Buenos Aires, Argentina and D Swift NOAA, Miami, Florida.

\section{UM-1620. Nr1}

$$
\begin{array}{r}
\mathbf{2 4 , 9 0 0} \\
\delta^{13} \mathrm{C}=+\mathbf{5 3 0} \\
-\mathbf{5 0 0} \\
\hline 53 \%
\end{array}
$$

Below ancient barrier sand and lagoonal mud (on shore) $\left(36^{\circ} 50^{\prime} 45^{\prime \prime}\right.$ S, $56^{\circ} 44^{\prime} 49^{\prime \prime} \mathrm{W}$ ).

\section{UM-1621. Nr2}

$$
\mathbf{5 2 0 0} \pm \mathbf{1 3 0}
$$

$\delta^{13} C=+1.37 \%$

Green clay below barrier (offshore) (36 $45^{\prime} 43^{\prime \prime}$ S, $\left.56^{\circ} 37^{\prime} 13^{\prime \prime} \mathrm{W}\right)$.

UM-1622. Nr3

Crest of sand ridge $\left(36^{\circ} 50^{\prime} 48^{\prime \prime} \mathrm{S}, 56^{\circ} 33^{\prime} 36^{\prime \prime} \mathrm{W}\right)$.

UM-1623. 3 bis

Crest of sand ridge $\left(36^{\circ} 50^{\prime} 48^{\prime \prime} \mathrm{S}, 56^{\circ} 33^{\prime} 36^{\prime \prime} \mathrm{W}\right)$.

$$
10,390 \pm 180
$$$$
\delta^{13} \mathrm{C}=+1.92 \%
$$

$11,610 \pm 140$ $\delta^{13} \mathrm{C}=+1.83 \%$

\section{Western Indian Ocean series}

D. India

Carbonate ooze coll in piston cores from Western Indian Ocean at $\left(9^{\circ} 28.4^{\prime} \mathrm{S}, 52^{\circ} 09.9^{\prime} \mathrm{E}\right)$ and $\left(9^{\circ} 33.2^{\prime} \mathrm{S}\right.$ and $\left.52^{\circ} 31.5^{\prime} \mathrm{E}\right)$ were dated to reconstruct submarine stratigraphy. Coll 1976 and subm 1978 by D Johnson, Woods Hole Oceanog Inst, Woods Hole, Massachusetts. 
UM-1546. AII93 11PC 44 to 50

UM-1547. AII93 FPC 37 to 43

$21,110 \pm 240$

UM-1548. AII93 11PC 72 to 78

$+1800$

$\mathbf{3 9 , 0 8 0}$

$-1520$

\section{Geomorphology of India series}

Two shell samples, UM-1533 from SE India $\left(8^{\circ} 10^{\prime} \mathrm{N}, 77^{\circ} 38^{\prime} \mathrm{E}\right)$ and UM-1534 from NW Sri Lanka $\left(8^{\circ} 15^{\circ} \mathrm{N}, 79^{\circ} 50^{\prime} \mathrm{E}\right)$ were dated to develop geomorphology and Quaternary history of area. Coll by P Deraniyagala and R Gardner; subm by R Gardner, Oxford Univ.

UM-1533. HSE/112

UM-1534. SL/1 $\mathbf{2 6 , 9 9 0} \pm \mathbf{5 0}$ $\delta^{13} C=-8.37 \%$ o

$2820 \pm 80$ $\delta^{13} C=-2.06 \%$

II. ARCHEOLOGIC SAMPLES

\section{Oleta River series}

\section{A. United States}

Shell and charcoal from Oleta $R$ archaeol site (8 Da 1024) North Miami Beach, Dade Co, Florida $\left(25^{\circ} 50^{\prime} 42^{\prime \prime} \mathrm{N}, 80^{\circ} 08^{\prime} 24^{\prime \prime} \mathrm{W}\right)$ was dated to determine period of occupation of site as well as sea-level change. Coll 1978 and subm by R Carr, Dade Co Hist Survey.

UM-1550. Shell FS-82

UM-1551. Charcoal FS-82

$$
\begin{array}{r}
\mathbf{2 1 1 0} \pm \mathbf{7 0} \\
\delta^{13} C=-2.51 \% 0 \\
\mathbf{2 1 0 0} \pm \mathbf{7 0} \\
\delta^{13} C=-25.06 \% \text { o }
\end{array}
$$

General Comment (DSI): UM-1550 and -1551 are 2 different materials dating same event and average as $2100 \pm 50$ using lab statistics.

UM-1549. Arch Creek FS-47

$1020 \pm 60$

Marine shell recovered from Arch Creek site, prehistoric midden in Dade Co, Florida $\left(25^{\circ} 08^{\prime} 17^{\prime \prime} \mathrm{N}, 80^{\circ} 10^{\prime} 55^{\prime \prime} \mathrm{W}\right)$ was dated to determine settlement pattern chronology in Dade Co. Coll and subm by $\mathrm{R}$ Carr, Dade Co Hist Survey, Miami, Florida.

\section{UM-1624. 8-Ma-64 F.S. \#50}

$$
\begin{array}{r}
4090 \pm 75 \\
\delta^{13} C=-25.79 \%
\end{array}
$$

Charcoal sample for dating mound construction from gray sand layer in aboriginal sand mound $\left(27^{\circ} 13^{\prime} 45^{\prime \prime} \mathrm{N}, 82^{\circ} 08^{\prime} 31^{\prime \prime} \mathrm{W}\right)$. Mound located in low pine palmetto flatlands in central Florida. Coll Dec 1978 and subm by R Willis, Florida State Mus, Gainesville. 
UM-1625. A-8376

$\delta^{13} \mathrm{C}=-24.65 \%$

Fragment of aboriginal dugout canoe (pine) from exposed mucky shore of sand bottom lake in central Florida $\left(29^{\circ} 40^{\prime} 0^{\prime \prime} \mathrm{N}, 81^{\circ} 55^{\prime} 30^{\prime \prime}\right.$ W). Dating for correlation of canoe styles with culture periods. This canoe resembles UM-1450 (R, 1979, v 21, p 297). Coll and subm 1978 by $\mathrm{R}$ Willis, Florida State Mus, Gainesville.

\section{REFERENCES}

Calvert, M, Rudolph, Kim, and Stipp, J J, 1978, University of Miami radiocarbon dates XII: Radiocarbon, v 20, p 274-282.

Introne, D S and Stipp, J J, University of Miami radiocarbon dates XV: Radiocarbon, v 21, p 291-297. 